\title{
A DOMINANCE THEOREM FOR PARTITIONED HERMITIAN MATRICES
}

\author{
BY \\ RUSSELL MERRIS( $\left.{ }^{1}\right)$
}

\begin{abstract}
Let $A=\left(A_{i j}\right)$ be a partitioned positive semidefinite hermitian matrix, where $A_{i j}$ is $n$-square, $1 \leqq i, j \leqq m$. A class of ordered pairs of functions $\left(f_{1}, f_{2}\right)$ is given such that $\left(f_{1}\left(A_{i j}\right)\right)-\left(f_{2}\left(A_{i j}\right)\right)$ is positive semidefinite hermitian. Applications are given.
\end{abstract}

1. Exordium. The field is the complex numbers. For square matrices, $A$, the notation $A \geqq 0$ means $A$ is positive semidefinite hermitian. If $A_{1}, A_{2}$, and $A_{1}-A_{2} \geqq 0$, write $A_{1} \geqq A_{2}$.

Let $A \geqq 0$ be $m n$-square, partitioned into $m^{2} n$-square submatrices:

$$
A=\left(A_{i j}\right), \quad 1 \leqq i, j \leqq m,
$$

where each $A_{i j}$ is $n$-square. A number of recent papers, [1], [5], [6], [7], [10], [11], [12], have dealt with the question: For what functions $f$ taking $n$-square matrices to $p$-square matrices is it the case that the $m p$-square matrix

$$
A_{f}=\left(f\left(A_{i j}\right)\right) \geqq 0 ?
$$

In this article, the following question is considered: For what functions $f_{1}, f_{2}$ taking $n$-square matrices to $p$-square matrices is it the case that

$$
\left(f_{1}\left(A_{i j}\right)\right) \geqq\left(f_{2}\left(A_{i j}\right)\right) ?
$$

In order to give one answer to this question, a large class of functions is defined. The class includes generalized matrix functions and generalized trace functions. Applications of the main result include dominance theorems for principal submatrices of associated matrices and inequalities for the functions themselves, some of which specialize to new inequalities for generalized matrix functions.

The organization of the paper is as follows: In $\$ 2$, the main results are detailed. $\S 3$ contains proofs of the more difficult results. In $\$ 4$, converses and extensions of the most important result are discussed.

Received by the editors December 7, 1970.

AMS 1970 subject classifications. Primary 15A15, 15A45, 15A57; Secondary $20 \mathrm{C15}$.

Key words and phrases. Generalized matrix function, Schur function, associated transformation, Kronecker product, Hadamard (Schur) product, (group) character, orthogonality relations.

(1) This work was done while the author was a National Academy of Sciences-National Research Council Postdoctoral Research Associate at the National Bureau of Standards, Washington, D. C. 20234. 
2. Main results and consequences. Let $G$ be a subgroup of the symmetric group $S_{n}$. Let $\lambda$ be a character on $G$. Let $X=\left(x_{i j}\right)$ be a generic $n$-square matrix. For $1 \leqq r \leqq n$, define

$$
e_{r}(X)=\sum_{\sigma \in G} \lambda(\sigma) E_{r}\left(x_{1 \sigma(1)}, \ldots, x_{n \sigma(n)}\right),
$$

where $E_{r}$ is the $r$ th elementary symmetric function. Of course, $e_{r}$ depends on $G$ and $\lambda$.

When $r=n, e_{n}=d_{G}^{\lambda}$, the generalized matrix function [8], [15]. (If $G=S_{n}$ and $\lambda=$ sgn, $d_{G}^{\lambda}=$ det. If $G=S_{n}$ and $\lambda=1, d_{G}^{\lambda}=$ per, the permanent. If $G=\{1\}$ and $\lambda=1$, $d_{G}^{\lambda}=\boldsymbol{h}$, the product of the main diagonal elements.)

When $r=1, e_{1}=t_{G}^{\lambda}$, the generalized trace function [11]. (If $G=\{1\}$ and $\lambda=1, t_{G}^{\lambda}$ is the trace. If $G$ is the group generated by the cycle $(1 \cdots n)$ and $\lambda=1, t_{G}^{\lambda}(X)=f(X)$, the sum of the entries of $X$.)

To make life easier, it will henceforth be assumed that $\lambda$ and all other characters mentioned are irreducible. For the aficionado, we will attempt to point out when this assumption is used and how it can be avoided.

2.1. THEOREM. Let $A \geqq 0$ be the mn-square partitioned matrix of (1). Then $A_{e_{r}}=\left(e_{r}\left(A_{i j}\right)\right) \geqq 0$. Moreover, the eigenvalues of $A_{e_{r}}$ lie in the interval $\left[\eta^{r} c, \mu^{r} c\right]$ where $c, \eta, \mu$ are, respectively, a nonnegative integer depending on $G$ and $\lambda$, the minimum and maximum eigenvalue of $A$.

The number, $c$, will be determined in the proof.

Theorem 2.1 has previously been proved when $r=n$ and $\lambda(1)=1$ [6], and when $r=1[11]$.

Now, let

$$
T(G, \lambda)=\frac{\lambda(1)}{g} \sum_{\sigma \in G} \lambda(\sigma) \sigma
$$

where $1 \in G$ is the identity element and $g$ is the order of $G$. With respect to the group algebra inner product which makes $S_{n}$ an orthonormal basis, the linear operator (left multiplication) $T(G, \lambda)$ is hermitian. Since $\lambda$ is irreducible, $T(G, \lambda)$ is idempotent and hence is an orthogonal projection.

2.2. THEOREM. Let $G_{i}, i=1,2$, be subgroups of $S_{n}$ of orders $g_{i}$ with irreducible characters $\lambda_{i}$. Let $r$ be fixed. Let

$$
f_{i}=\left(\lambda_{i}(1) / g_{i}\right) e^{i}, \quad i=1,2,
$$

where $e^{i}$ is that $e_{r}$ corresponding to $G_{i}$ and $\lambda_{i}$. If $T\left(G_{1}, \lambda_{1}\right) \geqq T\left(G_{2}, \lambda_{2}\right)$ then

$$
\left(f_{1}\left(\dot{A}_{i j}\right)\right) \geqq\left(f_{2}\left(A_{i j}\right)\right) \text {. }
$$

In what follows, let us write $\left(G_{1}, \lambda_{1}\right)=(G, \lambda)$ and $\left(G_{2}, \lambda_{2}\right)=(H, \chi)$. Since $T(G, \lambda)$ and $T(H, \chi)$ are orthogonal projections, $T(G, \lambda) \geqq T(H, \chi)$ if and only if $[4$, pp. 148-149]

$$
T(G, \lambda) T(H, \chi)=T(H, \chi) .
$$

Frequent use will be made of this fact. 
The next result gives a character theoretic equivalence of the condition $T(G, \lambda)$ $\geqq T(H, \chi)$.

2.3. THEOREM. When $\lambda$ and $\chi$ are irreducible, (5) holds if and only if both of the following conditions hold:

(*) $\sum \lambda(\sigma) \chi\left(\sigma^{-1} \pi\right)=(g / \lambda(1)) \chi(\pi)$,

$(* *) \sum \lambda(\tau \sigma) \chi\left(\sigma^{-1} \pi\right)=0$

for all $\pi \in H$ and $\tau \in G \backslash H$. Both sums are over $\sigma \in G \cap H$.

In case $G \subset H,\left({ }^{* *}\right)$ is vacuous and we have

2.4. Corollary. If $G \subset H$ then (5) holds if and only if $\chi \mid G=(\chi(1) / \lambda(1)) \lambda$, i.e., the restriction of $\chi$ to $G$ is a multiple of $\lambda$.

Corollary 2.4 contains the cases that $\chi \mid G$ is still irreducible, and that $G$ is contained in $H^{*}=\{\sigma \in H:|\chi(\sigma)|=\chi(1)\}, \lambda=\chi / \chi(1)$. In case $G$ is normal in $H$, Corollary 2.4 says (5) holds if and only if $\lambda$ is an irreducible component of $\chi \mid H$, invariant under conjugation by elements from $H$ [2, p. 53].

In the context of Corollary 2.4, when $\chi(1)=1$ and $r=n$, Williamson [16, Theorem 1] proved that the main diagonal elements of the left side of (4) dominate the corresponding elements on the right side (the case $m=1$ ). Williamson's result was extended in [13] to the case $\chi(1) \geqq 1$, and either $\chi \mid G=\lambda$ or $G \subset H^{*}$ and $\lambda=\chi / \chi(1)$.

2.5. Corollary. If $\lambda$ is linear (i.e., $\lambda(1)=1$ ) and if $(5)$ holds then $G \subset H$ and hence $G \subset H^{*}$ and $\chi \mid G=\chi(1) \lambda$.

Proof. If $G \notin H$, choose $\tau \in G \backslash H$. From (**) it follows that $\sum \lambda(\sigma) \chi\left(\sigma^{-1} \pi\right)=0$ for all $\pi \in H$. This contradicts $(*)$.

2.6. THEOREM. If $\{\sigma \in G: \lambda(\sigma) \neq 0\} \subset H \subset G$ and if $\chi \in \lambda \mid H$ is an irreducible component, then (5) holds.

Some results are now obtained by making special choices for $A, G, \lambda, H$ and $\chi$.

2.7. Corollary. Let $E_{r}(X)=E_{r}\left(x_{11}, \ldots, x_{n n}\right)$ for $X=\left(x_{i j}\right)$ a generic $n$-square matrix. Then

$$
\left(\boldsymbol{E}_{r}\left(A_{i j}\right)\right) \geqq(\chi(1) / h)\left(e_{r}\left(A_{i j}\right)\right)
$$

where $e_{r}$ corresponds to any subgroup $H$ of $S_{n}$ and irreducible character $\chi$. In particular,

$$
\left(h\left(A_{i j}\right)\right) \geqq(\chi(1) / h)\left(d_{H}^{\chi}\left(A_{i j}\right)\right) \quad \text { and } \quad\left(\operatorname{trace}\left(A_{i j}\right)\right) \geqq(\chi(1) / h)\left(t_{H}^{\chi}\left(A_{i j}\right)\right) .
$$

Proof. Theorem 2.2 and Corollary 2.4 .

2.8. Lemma [12]. Let $A_{1}, \ldots, A_{m}$ be $t \times n$ matrices. The mn-square partitioned matrix $A=\left(A_{i}^{*} A_{j}\right) \geqq 0$. 
Proof.

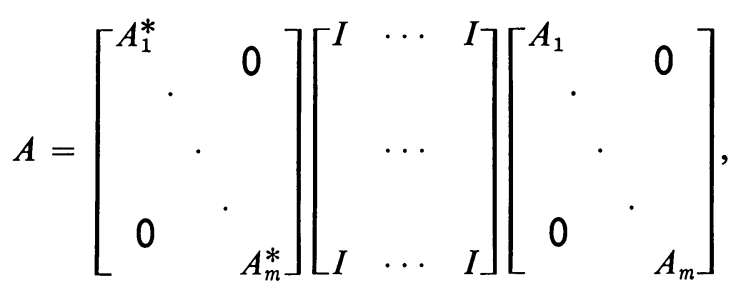

where $I$ is the $t$-square identity matrix.

One obtains a family of dominance theorems by combining 2.2 and 2.8. As an application of one of these theorems we have

2.9. Corollary. Let $A$ and $B$ be $t \times n$ matrices. Let $e^{i}, i=1,2$, be defined as in Theorem 2.2. Then

$$
c_{i}=e^{i}\left(A^{*} A\right) e^{i}\left(B^{*} B\right)-\left|e^{i}\left(A^{*} B\right)\right|^{2} \geqq 0 .
$$

Moreover, if $T\left(G_{1}, \lambda_{1}\right) \geqq T\left(G_{2}, \lambda_{2}\right)$ then

$$
\left(\lambda_{1}(1) / g_{1}\right)^{2} c_{1} \geqq\left(\lambda_{2}(1) / g_{2}\right)^{2} c_{2} .
$$

Proof. Apply 2.1 and 2.2 to 2.8 . Compare the leading $2 \times 2$ principal minors.

If $n=1,(6)$ is the Cauchy-Schwarz inequality. When $r=n$ and $\lambda_{i}(1)=1,(6)$ was proved in [8]. Freese [3] extended the result in [8] to the case $\lambda_{i}(1) \geqq 1$. When $r=1$, (6) was proved in [12].

Now, let $\Gamma_{n k}$ denote the set of functions from $\{1, \ldots, n\}$ to $\{1, \ldots, k\}$. If $B$ is a $k$-square matrix and if $\alpha, \beta \in \Gamma_{n k}$, we denote by $B[\alpha, \beta]$ the $n$-square matrix whose $i, j$ entry is the $\alpha(i), \beta(j)$ entry of $B$.

2.10. LemmA. Suppose $B \geqq 0$ is $k$-square. Let $\Gamma$ be a subset of $\Gamma_{n k}$ ordered arbitrarily. Let $m$ be the cardinality of $\Gamma$. Construct an mn-square partitioned matrix, $B(\Gamma)$, as follows: For $\alpha, \beta \in \Gamma$, the $\alpha, \beta$ block of $B(\Gamma)$ is $B[\alpha, \beta]$. Then $B(\Gamma) \geqq 0$.

Proof. Every principal submatrix of $B(\Gamma)$ is either permutation similar to a principal submatrix of $B$ or has two equal rows. Hence, every principal submatrix of $B(\Gamma)$ has nonnegative determinant. It is clear that $B(\Gamma)$ is hermitian when $B$ is.

Again, it is easy to see how to obtain a family of dominance theorems using 2.2 and 2.10. We will give some specific examples of these, but first we pause to provide some motivation. We define the associated matrix for $(G, \lambda)$. For this definition we assume that $\lambda(1)=1$. Associated matrices with $\lambda(1)>1$ have been treated by a number of authors. However, for our present purposes, the added generality does not seem worth the added effort.

We say two functions, $\alpha, \beta \in \Gamma_{n k}$ are equivalent $\bmod G$ if there is a $\sigma \in G\left(\subset S_{n}\right)$ such that $\alpha \sigma=\beta$. From each equivalence class $\bmod G$, choose the function which is 
lowest in lexicographic order. (Here we consider $\alpha=(\alpha(1), \ldots, \alpha(n)) \in \Gamma_{n k}$.) Denote the set of distinct representatives so chosen by $\Delta$. We let

$$
G_{\alpha}=\{\sigma \in G: \alpha \sigma=\alpha\}, \quad \alpha \in \Gamma_{n k}, \quad \text { and } \bar{\Delta}=\left\{\alpha \in \Delta: \lambda \mid G_{\alpha}=1\right\} .
$$

Order $\bar{\Delta}$ lexicographically. Let the cardinality of $\bar{\Delta}$ be $N$. Let $\nu(\alpha)$ be the order of $G_{\alpha}$.

If $C$ is $k$-square, the associated matrix for $(G, \lambda)$ of $C, K(C)$, is the $N$-square matrix whose $\alpha, \beta$ entry $(\alpha, \beta \in \bar{\Delta})$ is $(\nu(\alpha) \nu(\beta))^{-1 / 2} d_{G}^{\lambda} C^{T}[\beta, \alpha]$ where $C^{T}$ is the transpose of $C$. Perhaps the most interesting feature of these associated matrices is that $K\left(C_{1}\right) K\left(C_{2}\right)=K\left(C_{1} C_{2}\right)$.

2.11. Corollary (Marcus ANd Katz [6, Theorem 3]). Let $A \geqq 0$ be the $m n$ square partitioned matrix of (1). Then the $m N$-square partitioned matrix $\left(K\left(A_{i j}\right)\right) \geqq 0$.

Proof. Apply Lemma 2.10 to $A^{T}$. Apply Theorem 2.1 with $r=n$. Take the transpose. By a diagonal congruence $\left(\nu(\alpha)^{-1 / 2}, \alpha \in \bar{\Delta}\right.$ on the diagonal), $\left(K\left(A_{i j}\right)\right)$ is obtained.

Marcus and Katz used their result to prove that the block matrix

$$
\text { (trace } \left.K\left(A_{i j}\right)\right) \geqq 0 \text {, }
$$

from which they obtained a family of results. Using Corollary 2.7, (9) can be improved. For example, (trace $\left.K\left(A_{i j}\right)\right) \geqq N^{-1}\left(f\left(K\left(A_{i j}\right)\right)\right)$.

\subsection{Corollary. Let}

$$
\Gamma=\left\{\alpha \in \Gamma_{n k}: 1 \leqq \alpha(1)<\alpha(2)<\cdots<\alpha(n) \leqq k\right\} .
$$

Order $\Gamma$ lexicographically. Let $G=A_{n}$, the alternating group, and $\lambda=1$. If $B \geqq 0$ (and $k$-square) then

$$
2\left(d_{G}^{\lambda}\left(B^{T}[\beta, \alpha]\right)\right) \geqq\left(d\left(B^{T}[\beta, \alpha]\right)\right)=K,
$$

where $d=\operatorname{det}$ or $d=$ per.

If $d=\operatorname{det}, K$ is the $n$th compound of $B$ (the associated matrix for $\left.\left(S_{n}, \operatorname{sgn}\right)\right)$. If $d=$ per, $K$ is the principal submatrix of the $n$th power of $B$ (the associated matrix for $\left.\left(S_{n}, 1\right)\right)$ corresponding to the set $\Gamma$. The matrix on the left of $(11)$ is twice the principal submatrix corresponding to $\Gamma$, of the associated matrix for $\left(A_{n}, 1\right)$.

Corollary 2.12 is, of course, merely another example of Theorem 2 .

In view of Schur's inequalities [15] (e.g., per $B \geqq \operatorname{det} B$ for all $B \geqq 0$ ). It is tempting to conjecture that the principal submatrix of the $n$th power of $B$ corresponding to the $\Gamma$ of (10) be comparable to the $n$th compound. This is not the case as the following example shows:

$$
B=\left[\begin{array}{ll|ll}
2 & 0 & 1 & 1 \\
0 & 2 & 1 & 1 \\
\hline 1 & 1 & 2 & 0 \\
1 & 1 & 0 & 2
\end{array}\right] .
$$




\section{Proofs and byproducts.}

Proofs of 2.1 and 2.2. If $M=\left(m_{i j}\right)$ is $n$-square, let $K_{r}(M)$ be the $r$ th Kronecker power of $M$ (the $n^{r}$-square matrix whose $\alpha, \beta$ entry is $\prod_{i=1}^{r} m_{\alpha(i) \beta(i)}, \alpha, \beta \in \Gamma_{r n}$, where $\Gamma_{r n}$ is ordered lexicographically). If $N=\left(n_{i j}\right)$ is another $n$-square matrix, $M \circ N$ will denote the Hadamard or Schur product of $M$ and $N$, i.e., $M \circ N=\left(m_{i j} n_{i j}\right)$.

Let $\left\{Q(\sigma): \sigma \in S_{n}\right\}$ be the standard representation of $S_{n}$ by $n$-square permutation matrices; the $i, j$ element of $Q(\sigma)$ is $\delta_{i \sigma(j)}$. Let $D_{r n} \subset \Gamma_{r n}$ denote the subset of 1-1 functions. Let $Q_{r n} \subset D_{r n}$ be the subset of order preserving 1-1 functions. (The set $\Gamma$ in (10) is $Q_{n k}$.) Observe

$$
\begin{aligned}
r ! e_{r}(M) & =r ! \sum_{\sigma \in G} \lambda(\sigma) \sum_{\omega \in Q_{r n}} \prod_{t=1}^{r} m_{\omega(t) \sigma \omega(t)} \\
& =\sum_{\sigma \in G} \lambda(\sigma) \sum_{\omega \in D_{r n}} \prod_{t=1}^{r} m_{\omega(t) \sigma \omega(t)} \\
& =\sum_{\sigma \in G} \lambda(\sigma) \sum_{\omega, v \in D_{r n}} \prod_{t=1}^{r}\left(\delta_{v(t) \sigma \omega(t)} m_{\omega(t) v(t)}\right) \\
& =\sum_{\sigma \in G} \lambda(\sigma) \sum_{\omega, v \in D_{r n}} \prod_{t=1}^{r}\left(Q\left(\sigma^{-1}\right)_{\omega(t) v(t)} m_{\omega(t) v(t)}\right) \\
& =\sum_{\sigma \in G} \lambda(\sigma) \sum_{\omega, v \in D_{r n}}\left(K_{r}\left(Q\left(\sigma^{-1}\right)\right)\right)_{\omega \nu}\left(K_{r}(M)\right)_{\omega v} \\
& =f\left(C_{r}^{\prime}(G, \lambda) \circ K_{r}^{\prime}(M)\right),
\end{aligned}
$$

where (recall) $\boldsymbol{f}$ is the function which sums the elements of the matrix it sees; $C_{r}(G, \lambda)=\sum_{\sigma \in G} \lambda(\sigma) K_{r}\left(Q\left(\sigma^{-1}\right)\right)$; and primes indicate the principal submatrix corresponding to $D_{r n}$.

It is trivial to see that the block matrix $\left(K_{r}\left(A_{i j}\right)\right)$ is a principal submatrix of $K_{r}(A)$, and that $\left(K_{r}^{\prime}\left(A_{i j}\right)\right)$ is a principal submatrix of $\left(K_{r}\left(A_{i j}\right)\right)$. Hence, $\left(K_{r}^{\prime}\left(A_{i j}\right)\right) \geqq 0$ with eigenvalues contained in $\left[\eta^{r}, \mu^{r}\right]$, the interval determined by the minimum and maximum eigenvalues of $K_{r}(A)$.

Let $\psi_{r}$ be the function from $S_{n}$ to the $n^{r}$-square complex matrices defined by

$$
\psi_{r}(\sigma)=K_{r}(Q(\sigma)) \text {. }
$$

Then $\psi_{r}$ is a representation of $S_{n}$. It follows that $(\lambda(1) / g) C_{r}(G, \lambda)$ is hermitian (equal to its conjugate transpose) and idempotent. Hence $C_{r}(G, \lambda) \geqq 0$. Thus the Kronecker product $J \otimes C_{r}^{\prime}(G, \lambda) \geqq 0$, where $J$ is the $m$-square matrix each of whose entries is 1 . Therefore the $\left(m\left(r !\left(\begin{array}{l}n \\ r\end{array}\right)\right)\right)$-square block matrix

$$
\left(J \otimes C_{r}^{\prime}(G, \lambda)\right) \circ\left(K_{r}^{\prime}\left(A_{i j}\right)\right)=\left(C_{r}^{\prime}(G, \lambda) \circ K_{r}^{\prime}\left(A_{i j}\right)\right) \geqq 0 .
$$

We now need a special case of Theorem 2.1:

3.1. LEMMA. Let $\boldsymbol{f}$ be the function which sums the elements of the matrix it sees. If $A=\left(A_{i j}\right) \geqq 0$ then $A_{f}=\left(f\left(A_{i j}\right)\right) \geqq 0$. Moreover, if $M=\left(M_{i j}\right) \geqq 0$ has the same size and partitioning as $A$, and if $A \geqq M$, then $A_{f} \geqq M_{f}$. 
Proofs of this lemma are contained in [10] and [11]. However, the following proof is very short:

Let $C$ be the $m n \times m$ matrix whose $i$ th column contains a 1 in each of positions $n(i-1)+1, \ldots, n i$, and zeros elsewhere. Then $A_{f}=C^{*} A C$.

This proof also works for the case that $A_{i j}$ is $n_{i} \times n_{j}, n_{i}$ not necessarily equal to $n_{j}$.

In view of (14), to prove that $A_{e_{r}} \geqq 0$, it remains to apply Lemma 3.1 to (16) and divide by $r$ !.

We now supply the bounds for the eigenvalues of $A_{e_{r}}$. Let $M$ be the matrix of Lemma $3.1(A \geqq M)$. It is not difficult to prove that $K_{r}(A) \geqq K_{r}(M)$ (see, for example, [9]). Hence,

$$
\left(K_{r}^{\prime}\left(A_{i j}\right)\right) \geqq\left(K_{r}^{\prime}\left(M_{i j}\right)\right) .
$$

It follows from (16) and (17) that

$$
\left(C_{r}^{\prime}(G, \lambda) \circ K_{r}^{\prime}\left(A_{i j}\right)\right) \geqq\left(C_{r}^{\prime}(G, \lambda) \circ K_{r}^{\prime}\left(M_{i j}\right)\right) .
$$

Take $M=\eta I$ where $I$ is the $m n$-square identity and apply Lemma 3.1 to (18). We obtain that $\left(e_{r}\left(A_{i j}\right)\right)$ dominates the $m$-square scalar matrix each of whose diagonal entries is $\eta^{r} c$ where

$$
\begin{aligned}
c & =(r !)^{-1} \operatorname{trace} C_{r}^{\prime}(G, \lambda) \\
& =(r !)^{-1} \sum_{\omega \in D_{r n}} \sum_{\sigma \in G} \lambda(\sigma) \prod_{t=1}^{r} \delta_{\omega(t) \sigma \omega(t)} \\
& =(r !)^{-1} \sum_{\omega \in D_{r n}} \sum_{\sigma \in G(\omega)} \lambda(\sigma) \quad \text { where } G(\omega)=\{\sigma \in G: \sigma \omega=\omega\} .
\end{aligned}
$$

(The notation $G(\omega)$ should not be confused with $G_{\omega}$.) Let $g(\omega)$ be the order of $G(\omega)$ and $(\lambda \mid G(\omega), 1)$ be the number of occurrences of the principal representation in the restriction of $\lambda$ to the group $G(\omega)$. Then we have

$$
c=\sum_{\omega \in Q_{r n}} \sum_{\sigma \in G(\omega)} \lambda(\sigma)=\sum_{\omega \in Q_{r n}} g(\omega)(\lambda \mid G(\omega), 1),
$$

certainly a nonnegative integer. The upper bound for the eigenvalues of $A_{e_{r}}$ is obtained in the same way.

To prove Theorem 2.2, extend $\psi_{r}$ (see (15)) linearly to a transformation from the $S_{n}$-algebra. (In general, if $G$ is a group, by the $G$-algebra, we mean the group algebra of $G$ over the complex numbers.) Then $\psi_{r}$ is a homomorphism from the $S_{n}$-algebra to the algebra of $n^{r}$-square matrices. Therefore, (5) implies

$$
(\lambda(1) / g) C_{r}(G, \lambda) \geqq(\chi(1) / h) C_{r}(H, \chi) .
$$

(Indeed, taking (21) as our hypothesis in place of $T(G, \lambda) \geqq T(H, \chi)$, we could avoid the assumption of irreducibility and obtain a stronger theorem.) A combination of (14), (16), (21) and Lemma 3.1 yields the result. 
Proof of Theorem 2.3. The idea of the proof is to consider (5) as an equation in the group algebra and to take advantage of the fact that $\left\{\sigma \in S_{n}\right\}$ is a basis. Thus

$$
T(G, \lambda) T(H, \chi)=\frac{\lambda(1) \chi(1)}{g h} \sum_{\sigma \in G ; \pi \in H} \lambda(\sigma) \chi(\pi) \sigma \pi .
$$

Now, $\sigma \pi \in H$ if and only if $\sigma \in H$. Therefore, (22) breaks naturally into two equations:

$$
\begin{aligned}
& \left({ }^{\prime}\right)^{\prime} \frac{\lambda(1) \chi(1)}{g h} \sum_{\sigma \in G \cap H ; \pi \in H} \lambda(\sigma) \chi(\pi) \sigma \pi=T(H, \chi) \text { and } \\
& \left(*^{* *}\right)^{\prime} \sum_{\sigma \in G \backslash H ; \pi \in H} \lambda(\sigma) \chi(\pi) \sigma \pi=0 .
\end{aligned}
$$

By a change of variables, $\left({ }^{*}\right)^{\prime}$ becomes

$$
\frac{\lambda(1) \chi(1)}{g h} \sum_{\sigma \in G \cap H ; \pi \in H} \lambda(\sigma) \chi\left(\sigma^{-1} \pi\right) \pi=T(H, \chi)
$$

or

$$
\frac{\lambda(1)}{g} \sum_{\sigma \in G \cap H} \lambda(\sigma) \chi\left(\sigma^{-1} \pi\right)=\chi(\pi) \text { for all } \pi \in H
$$

In (23), $\sigma_{1} \pi_{1}=\sigma_{2} \pi_{2}$ if and only if $\sigma_{1}=\sigma_{2} \tau$ and $\pi_{1}=\tau^{-1} \pi_{2}$ for some $\tau \in G \cap H$. Therefore, the coefficient of any particular $\sigma \pi$ in (23) is

$$
\sum_{\tau \in G \cap H} \lambda(\sigma \tau) \chi\left(\tau^{-1} \pi\right)
$$

But, (24) must be zero for every $\sigma \in G \backslash H$ and $\pi \in H$. Change $\sigma$ to $\tau$ and $\tau$ to $\sigma$ in (24) to obtain $\left({ }^{* *}\right)$.

Proof of Corollary 2.4. Set $\pi=1$ in $\left(^{*}\right)$ to obtain

$$
(\chi \mid G, \lambda)=g^{-1} \sum_{\sigma \in G} \lambda(\sigma) \chi\left(\sigma^{-1}\right)=\chi(1) / \lambda(1)
$$

Hence,

$$
\chi \mid G=(\chi(1) / \lambda(1)) \lambda
$$

Conversely, assume (25) holds. One can easily show that $T(G, \lambda)$ and $T(H, \chi)$ commute. (Indeed, $T(H, \chi)$ is in the center of the $H$-algebra to which $T(G, \lambda)$ belongs.) Therefore, their product is an orthogonal projection. But,

$$
T(H, \chi)(T(G, \lambda) T(H, \chi))=T(G, \lambda) T(H, \chi) .
$$

Hence, $T(H, \chi) \geqq T(G, \lambda) T(H, \chi)$. It remains to prove

$$
\operatorname{rank} T(H, \chi)=\operatorname{rank} T(G, \lambda) T(H, \chi) .
$$


But, the rank of an orthogonal projection is just its trace. So, let $\xi$ be the character of the regular representation of $H$. Then, since $\xi(\sigma)=0$ if $\sigma \neq 1$,

$$
\begin{aligned}
\operatorname{trace} T(H, \chi)= & (\chi(1) / h) \sum_{\sigma \in H} \chi(\sigma) \xi(\sigma)=\chi(1)^{2} . \\
\operatorname{trace} T(G, \lambda) T(H, \chi) & =\frac{\lambda(1) \chi(1)}{g h} \sum_{\sigma \in G} \lambda(\sigma) \sum_{\pi \in H} \chi(\pi) \xi(\sigma \pi) \\
& =\frac{\lambda(1) \chi(1)}{g} \sum_{\sigma \in G} \lambda(\sigma) \chi\left(\sigma^{-1}\right) \\
& =\frac{\chi(1)^{2}}{g} \sum_{\sigma \in G} \lambda(\sigma) \lambda\left(\sigma^{-1}\right) \quad \text { from (25) } \\
& =\chi(1)^{2} . \quad \text { Q.E.D. }
\end{aligned}
$$

As a bonus, we have proved the following extension of a standard orthogonality relation.

3.2. THEOREM. Let $G$ be a subgroup of $H$. Let $\lambda$ and $\chi$ be, respectively, irreducible characters on $G$ and $H$. Suppose that $\chi \mid G=(\chi(1) / \lambda(1)) \lambda$. (In particular, this will be true if $G$ is normal in $H$ and if $\lambda \in \chi \mid G$ is invariant under conjugation by elements of $H$, or if $G$ is an arbitrary subgroup of $H$ such that $\chi \mid G$ is irreducible on $G$.) Then

for all $\pi \in H$.

$$
\sum_{\sigma \in G} \lambda(\sigma) \chi\left(\sigma^{-1} \pi\right)=\frac{g}{\lambda(1)} \chi(\pi)
$$

Theorem 3.2 can also be proved directly using the usual methods [2, pp. 32-33] or [14, pp. 15-16]. (Indeed, a slicker proof of the converse in Corollary 2.4 would be to apply Theorem 3.2 directly to $(25)$, obtaining $\left({ }^{*}\right)$.)

Proof of Theorem 2.6.

$$
\begin{aligned}
T(G, \lambda) T(H, \chi) & =\frac{\lambda(1) \chi(1)}{g h} \sum_{\sigma \in G ; \pi \in H} \lambda(\sigma) \chi(\pi) \sigma \pi \\
& =\frac{\lambda(1) \chi(1)}{g h} \sum_{\sigma, \pi \in H} \lambda(\sigma) \chi(\pi) \sigma \pi \\
& =\frac{\lambda(1)}{g} \sum_{\tau \in H}\left[\frac{\chi(1)}{h} \sum_{\sigma \in H} \lambda(\sigma) \chi\left(\sigma^{-1} \tau\right)\right] \tau \\
& =\frac{\lambda(1)}{g}(\lambda \mid H, \chi) \sum_{\tau \in H} \chi(\tau) \tau
\end{aligned}
$$

by the orthogonality relations ( $\chi$ is irreducible). Since $T(G, \lambda)$ and $T(H, \chi)$ commute, their product, (26), is a projection. It follows, since we have assumed $(\lambda \mid H, \chi) \neq 0$, that $(26)$ is $T(H, \chi)$, i.e., that

$$
(\lambda(1) / g)(\lambda \mid H, \chi)=\chi(1) / h
$$

and we are finished. 
In the context of Theorem 2.6 (when $G \neq H$ ), we have shown that $\lambda \mid H$ always reduces; if not, $(\lambda \mid H, \chi)=1$ and $\lambda(1)=\chi(1)$ but $g \neq h$, contradicting (27).

\section{Converses and extensions.}

Extensions of 2.1 and 2.2. Consider any $\bar{\Delta}$ set of the type (8) where we want the notation such that $\bar{\Delta} \subset \Gamma_{r n}$. The $r$ th Schur function of $y=\left(y_{1}, \ldots, y_{n}\right)$ corresponding to $\bar{\Delta}$ is defined by

$$
F_{r}(y)=\sum_{\omega \in \bar{\Delta}} \prod_{t=1}^{r} y_{\omega(t)}
$$

(For example, if $G=S_{r}$ and $\lambda=\operatorname{sgn}, \bar{\Delta}=Q_{r n}$. Thus, the Schur function corresponding to this $\bar{\Delta}$ is $E_{r}$, the $r$ th elementary symmetric function.)

Now, let $H$ be a subgroup of $S_{n}$ with character $\chi$. Given a fixed Schur function, $F_{r}$, define

$$
s_{r}(X)=\sum_{\sigma \in H} \chi(\sigma) F_{r}\left(x_{1 \sigma(1)}, \ldots, x_{n \sigma(n)}\right)
$$

where $X=\left(x_{i j}\right)$ is a generic $n$-square matrix. Of course, $s_{r}$ depends on $H, \chi, G$, and $\lambda$.

One can replace $e_{r}$ in 2.1 and 2.2 with $s_{r}$ and obtain exactly the same theorems. The proofs proceed analagously except that some additions are needed to deal with the more complicated combinatorial structure of the general $\bar{\Delta}$ set.

Indeed, one could extend 2.1 and 2.2 to even more general functions. What is needed from the indices of summation is that the set have enough symmetry to permit the analog of (12)-(13). For example, if $n>1$ then

$$
\{(i, \ldots, i): 1 \leqq i \leqq n\} \subset \Gamma_{r n}
$$

is such an index set which is not a $\bar{\Delta}$ set. In this case, the analog of (2) is

$$
\sum_{\sigma \in G} \lambda(\sigma) \sum_{t=1}^{n} x_{t \sigma(t)}^{r}
$$

Before discussing a converse of Theorem 2.2, we illustrate some of the difficulties with an example. Let $G=S_{3}, \lambda_{1}=1, \lambda_{2}=$ sgn. Then the matrix representation of $T\left(G, \lambda_{1}\right)-T\left(G, \lambda_{2}\right)$ with respect to the ordered basis $\{1,(12),(13),(23),(123),(132)\}$ is

$$
\frac{1}{3}\left[\begin{array}{llllll}
0 & 1 & 1 & 1 & 0 & 0 \\
1 & 0 & 0 & 0 & 1 & 1 \\
1 & 0 & 0 & 0 & 1 & 1 \\
1 & 0 & 0 & 0 & 1 & 1 \\
0 & 1 & 1 & 1 & 0 & 0 \\
0 & 1 & 1 & 1 & 0 & 0
\end{array}\right],
$$


clearly not positive semidefinite. However,

$$
C_{1}\left(G, \lambda_{1}\right)-C_{1}\left(G, \lambda_{2}\right)=\frac{1}{3}\left[\begin{array}{lll}
1 & 1 & 1 \\
1 & 1 & 1 \\
1 & 1 & 1
\end{array}\right] .
$$

This shows that the following would-be converse to Theorem 2.2 fails: Let $f_{1}, f_{2}$ be as in Theorem 2.2. Suppose that $f_{1}(B) \geqq f_{2}(B)$ for all $n$-square $B \geqq 0$. Then $T\left(G_{1}, \lambda_{1}\right)$ $\geqq T\left(G_{2}, \lambda_{2}\right)$. However, we do have

4.1. THEOREM. Let $f_{1}$ and $f_{2}$ be as in Theorem 2.2 with $r=1$. If $f_{1}(B) \geqq f_{2}(B)$ for all $n$-square $B \geqq 0$, then

$$
\left(\lambda_{1}(1) / g_{1}\right) C_{1}\left(G_{1}, \lambda_{1}\right) \geqq\left(\lambda_{2}(1) / g_{2}\right) C_{1}\left(G_{2}, \lambda_{2}\right) .
$$

Proof. For $i=1,2$, let

$$
T_{i}=\left(\lambda_{i}(1) / g_{i}\right) C_{1}\left(G_{i}, \lambda_{i}\right)^{T}=\left(\lambda_{i}(1) / g_{i}\right) \sum_{\sigma \in G_{i}} \lambda_{i}(\sigma) Q(\sigma) .
$$

It is an easy computation to show that $f_{i}(B)=\operatorname{trace}\left(T_{i} B\right)$. Assume trace $\left(T_{1} M^{*} M\right)$ $\geqq \operatorname{trace}\left(T_{2} M^{*} M\right)$ for all $n$-square $M$. Let $U$ be unitary such that $U^{*}\left(T_{1}-T_{2}\right) U$ is diagonal. Then

trace $\left(\left(T_{1}-T_{2}\right) M^{*} M\right) \geqq 0$ for all $M$

if and only if

trace $\left(U^{*}\left(T_{1}-T_{2}\right) U U^{*} M^{*} M U\right) \geqq 0$ for all $M$

if and only if

trace $\left(U^{*}\left(T_{1}-T_{2}\right) U M^{*} M\right) \geqq 0$ for all $M$

if and only if

the eigenvalues of $T_{1}-T_{2}$ are all nonnegative

if and only if

$T_{1} \geqq T_{2}$.

Theorem 4.1 is not true when $r=1$ is replaced with $r=n$. I do not know about $1<r<n$.

\section{REFERENCES}

1. John de Pillis, Transformations on partitioned matrices, Duke Math. J. 36 (1969), 511-515.

2. Walter Feit, Characters of finite groups, Benjamin, New York, 1967. MR 36 \#2715.

3. Ralph Freese, Inequalities for generalized matrix functions based on arbitrary characters, Linear Algebra Appl. (to appear).

4. P. R. Halmos, Finite dimensional vector spaces, 2nd ed., Van Nostrand, Princeton, N. J., 1958. MR 19, 725.

5. Charles Löwner, Some theorems on positive matrices (unpublished).

6. Marvin Marcus and Susan M. Katz, Matrices of Schur functions, Duke Math. J. 36 (1969), 343-352. MR 39 \#4182.

7. Marvin Marcus and Nisar A. Khan, A note on the Hadamard product, Canad. Math. Bull. 2 (1959), 81-83. MR 21 \#4166. 
8. Marvin Marcus and Henryk Minc, Generalized matrix functions, Trans. Amer. Math. Soc. 116 (1965), 316-329. MR 33 \#2655.

9. Marvin Marcus and Paul Nikolai, Inequalities for some monotone matrix functions, Canad. J. Math. 21 (1969), 485-494. MR 38 \#5815.

10. Marvin Marcus and William Watkins, Partitioned hermitian matrices, Duke Math. J. 38 (1971), 237-249.

11. Russell Merris, Trace functions. I, Duke Math. J. (to appear).

12. — Partitioned hermitian matrices, J. Res. Nat. Bur. Standards Sect. B 74B (1970), $45-46$.

13. Russell Merris and William Watkins, Group algebra ideals and symmetry classes of tensors (to appear).

14. Morris Newman, Matrix representations of groups, Nat. Bur. Standards Appl. Math. Series, no. 60, Superintendent of Documents, U.S. Government Printing Office, Washington, D.C., 1968. MR 40 \#250.

15. I. Schur, Uber endliche Gruppen und hermitesche Formen, Math. Z. 1 (1918), 184-207.

16. S. G. Williamson, On a class of combinatorial inequalities, J. Combinatorial Theory 6 (1969), 359-369. MR 39 \#233.

National Bureau of Standards, Washington, D.C. 20234

Current address: Department of Mathematics, California State College, Hayward, California 94542 